\title{
The Energy Loss Due to Interconnections in Paralleled Cell Configurations of Lithium-Ion Batteries in Electric Vehicles
}

\author{
Mohamed Ahmeid \\ School of Engineering \\ Newcastle University \\ Newcastle upon Tyne, UK \\ Mohamed.Ahmeid1@ncl.ac.uk \\ Simon Lambert \\ School of Engineering \\ Newcastle University \\ Newcastle upon Tyne, UK \\ Simon.Lambert@ncl.ac.uk
}

\author{
Musbahu Muhammad \\ School of Engineering \\ Newcastle University \\ Newcastle upon Tyne, UK \\ Musbahu.Muhammad@ncl.ac.uk \\ Pierrot Attidekou \\ School of Engineering \\ Newcastle University \\ Newcastle upon Tyne, UK \\ Pierrot.Attidekou@ncl.ac.uk
}

\author{
Zoran Milojevic \\ School of Engineering \\ Newcastle University \\ Newcastle upon Tyne, UK \\ Zoran.Milojevic@ncl.ac.uk
}

\begin{abstract}
In Electric vehicles, the Li-ion battery reaches its end of life when the capacity is decreased to $80 \%$ of the initial rated capacity. However, a battery with only $20 \%$ used capacity does not mean the battery cannot be used in a secondary application with less current demand, in a controlled and secure environment. This necessitates a comprehensive understanding of the configuration of the end of life (EoL) batteries in module and pack level, in terms of inconsistencies in capacity and impedance of the cells forming the module and hence the battery pack. Accordingly, a safer and longer second life use can be granted. This paper investigates the impact of parallel connection on the impedance and capacity of four, pouch lithium-ion cells forming a battery module in $2 \mathrm{P} 2 \mathrm{~S}$ configuration. The energy storage capacity and the AC impedance of each parallel pair and individual cells are recorded and compared. The results highlight that the capacity loss due to the parallel connection is $6 \%$ less than the sum of the individual capacity for each cell. With the help of a developed equivalent circuit model, the ohmic resistance of the pair and cell is estimated to demonstrate the contribution of interconnections in increasing the total impedance and hence the perceived loss of capacity in the parallel connection.
\end{abstract}

Keywords-Li-ion battery, electric vehicles, second life, module, capacity, impedance.

\section{INTRODUCTION}

In recent years the demand for using lithium-ion batteries in many applications has grown considerably. Among these applications, the size of the electric vehicle (EV) fleet has substantially increased due to their zero-emission nature which reduces the contribution to climate change and pollution[1]. With a greater number of EVs entering the market, the number of $\mathrm{Li}$-ion batteries entering the recycling and waste streams at their end of life (EoL) is expected to surge. Consequently, the capability to recycle, refurbish, and reuse those batteries is becoming vital to boost the circular economy and reduce ecological damage. To effectively reuse the lithium-ion battery in a second life application, such as an off-highway energy storage system, testing and analysing its performance in terms of available capacity and power is essential. Based on the application requirements, the lithiumion batteries can be organised in series to increase the voltage level and in a parallel to increase the current and the available capacity or in a combination of both of these (as is commonly done in EVs)[2]. In hybrid and electric vehicles, the battery pack is composed of many single Lithium-ion cells electrically connected together to fulfil the requirements for power and energy. Generally, there are three main types of battery cells employed in automotive applications. For example, the Nissan Leaf $24 \mathrm{kWh}$ battery pack consists of 33 Ah pouch cells, with 2 in parallel and 96 in series. Contrariwise, the Tesla Model S $85 \mathrm{kWh}$ battery pack uses 743.1 Ah cylindrical cells to form a parallel unit, and 96 of these units in series [3]. In the BMW i3 $42.2 \mathrm{kWh}$ battery, the battery pack consists of 12 prismatic cells in parallel and 8 in series [4]. Because of the physical design and size, the prismatic and pouch cells allow economically feasible second use applications where the packs can be repurposed at the pack or module level [5]. In the module level, the parallel connection can be made from 12 cells such as in BMW i3 or 2 pouch cells in Nissan Leaf. Regardless of the advantages of connecting Li-ion cells in parallel, there are some challenges reported in the literature related to the electrical, mechanical and thermal integration of cells into packs [6]. In [1] the authors investigated different scenarios in parallel connection which can degrade the overall performance of battery pack, such as, manufacturing tolerance, interconnect resistance, and ambient temperature. The results presented highlight that the cell will be aged faster as the length of the parallel connection increases. Michael et al. [7] presented a detailed examination of all occurring critical parameter variations within parallel cells. After characterisation measurements, it was quantified that the maximum inhomogeneity in capacities and resistances of parallel couples within a retired battery pack are $2.23 \%$ and $10 \%$ respectively. Gregory et al.[8] stated that the difference in current flow across the battery modules occurs mainly due to the interconnection resistances between highly paralleled cells irrespective of the variations in impedance between cells. Therefore, it is of high interest to understand the interaction between these parameters in particular for retired EV batteries in order to forecast their performance in second life applications and to mitigate any safety concerns. The objective of this paper is to demonstrate the hidden contribution of interconnections in the loss of capacity in paralleled cells. This study comprises disassembly of a battery module from a retired Nissan Leaf $24 \mathrm{kWh}$ battery pack. The discharge capacity and the impedance for a module and cell level are measured to show the correlation between the ohmic resistance and capacity in parallel connection. The overall output of this work is expected to highlight the importance of improving welding and interconnections techniques for pouch cells to utilise the most available capacity within the pack. 


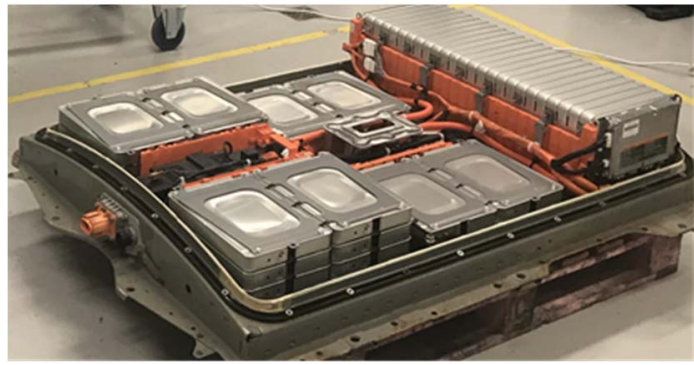

Fig. 1. $24 \mathrm{kWh}$ Nissan Leaf battery pack.

\section{EXPERIMENTAL PROCEDURE}

In this study, the battery module used consists of four pouch cells connected in a two-parallel, two-series arrangement forming two pairs. This module is randomly selected from a retired $24 \mathrm{kWh}$ Nissan Leaf battery pack (Fig.1) to study the behaviour of parallel connected cells within one module. Fig. 2 shows the module where the pouch cells' terminals are welded to a coper bus bars to form the electrical connections. Each module has three terminals red, white and black (RWB) which form the positive and negative terminals of the module. The centre tap white is either positive or negative terminal relative to the two by two arrangement. The maximum voltage of each pair is $4.2 \mathrm{~V}$. Prior to testing and disassembly of the battery pack, the data recorded by the battery management system (BMS) regarding the energy fade and hence the end of life decision is collected. This indicates that the state of health $(\mathrm{SoH}$, defined as the ratio of the remaining capacity to the design capacity) of the battery pack is $76.96 \%$ which equates to a two-cell-in-parallel capacity of $50.49 \mathrm{Ah}$. This is expected to be the actual capacity of the weakest cell pair among the 96 batteries connected in series in the pack and is below the common industry standard for EoL which is an $\mathrm{SoH}$ of $80 \%$. For experimental validation, the testing platform mainly consisted of an HCP-1005 potentiostat from bio-logic and an environmental chamber. The potentiostat is controlled by ECLab software that performs the capacity and impedance tests. During the experiment, the temperature is kept at $25 \mathrm{C}$. For the capacity test, the Constant Current-Constant Voltage (CC$\mathrm{CV}$ ) charging protocol is used to charge the pair to $100 \%$ SoC, during the CC stage the cell is charged at $1 \mathrm{C}$ current until the voltage rises to $4.2 \mathrm{~V}$. Subsequently, the CV phase is involved to complete the charging until the current fell to $\mathrm{C} / 20$ (3.3A). After 30 mins as resting period, the CC-CV is repeated to discharge the pair to cutoff voltage $(2.7 \mathrm{~V})$. Complementary to the capacity test, the impedance measurements were taken at different SoC $(100 \%, 80 \%$, $60 \%, 40 \%$, and $20 \%$ ) at a controlled temperature of $25{ }^{\circ} \mathrm{C}$. The Electrochemical Impedance Spectroscopy (EIS) test is performed under a potentiostatic mode from $1 \mathrm{kHz}$ to 10 $\mathrm{mHz}$, with a sine amplitude of $7.1 \mathrm{mV}$ for each SoC when the pair reaches its equilibrium state. Following, the module is dismantled separating assembly pieces and bus bars from the pouch cells then, the same electrical cells, tests are repeated for each pouch cell individually under the same conditions.

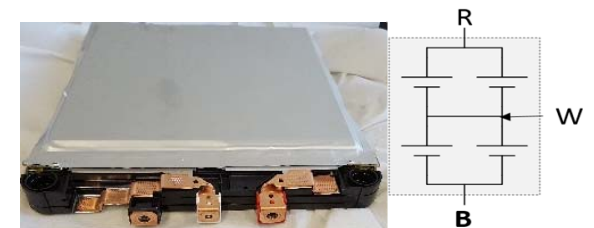

Fig. 2. Nissan Leaf module and circuit diagram.

\section{RESULTS AND DISCUSSION}

Fig. $3 a$ and $b$ illustrate the discharge capacity of the two pairs in the Nissan Leaf module. Although both parallel cells forming Pair 2 have an identical capacity $C_{3}=C_{4}=27.8 \mathrm{Ah}$, the total capacity measured when connected in parallel is less than the sum of the individual cells (51.8 Ah). Similarly, the actual capacity of Pair 1 is 51.3 Ah while both cells have a slightly different in capacities of $C_{1}=27.8 \mathrm{Ah}$ and $C_{2}=27.5$ Ah when measured separately. This drop in the total capacity 3.8 $\mathrm{Ah}$ in Pair 1 and $4 \mathrm{Ah}$ in Pair2 indicates that the connection resistance within the parallel unit is not negligible. Due to this loss, the entire battery pack performance is affected as the estimated $\mathrm{SoH}$ based on the measured capacity of Pair 1 is $78.3 \%$ while the theoretical SoH based on actual capacities of both cells is $84.4 \%$ which means $4 \%$ higher than the EoL limit reducing the useful life of the pack in its primary application (EV). Similarly, the recycling decision after the battery used in a second life application will be taken while the pouch cells still possess some useable energy that allows the module to serve for longer cycle life.
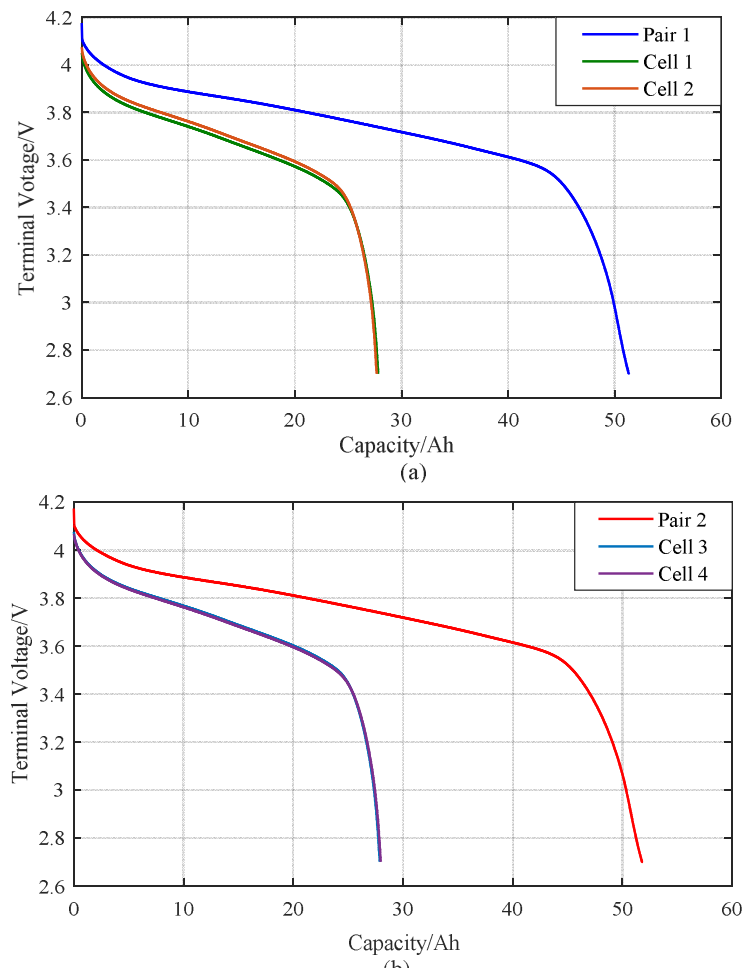

Fig. 3. The discharge capacity (a) pair1, cell 1, and cell 2. (b) Pair 2 cell 3 , and cell 4. 

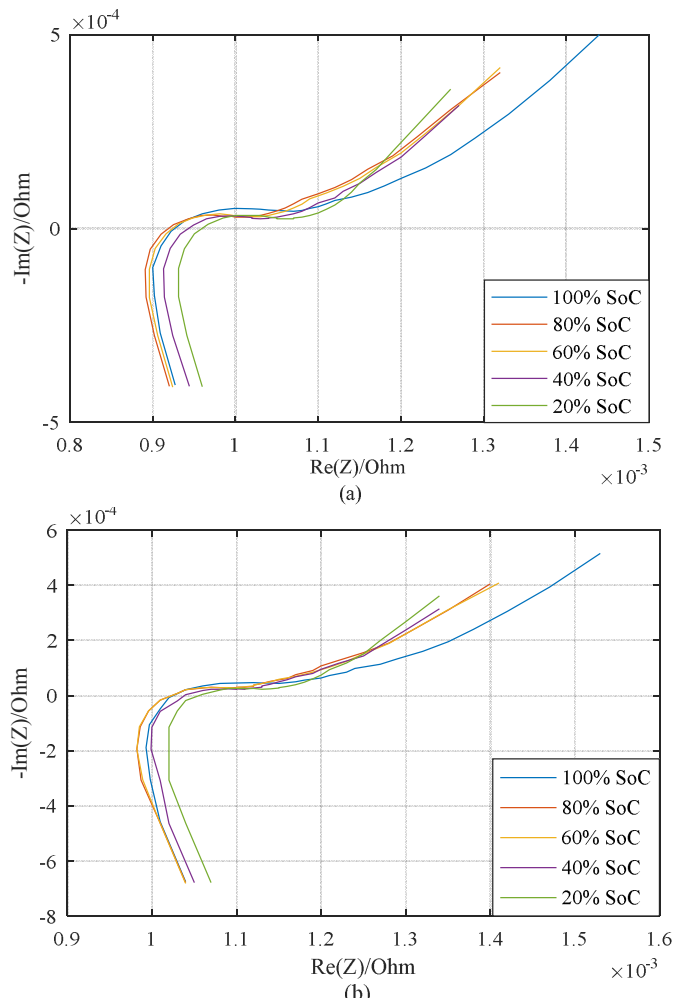

Fig. 4. Nyquist plot for parallel cells in Leaf module at $\mathrm{SoC}$ values from $20 \% \sim 100 \%$ (a) Pair 1. (b) Pair2.

To further investigate the impact of interconnections on the total impedance of parallel connection, both pairs and individual cells are subjected to the Electrochemical Impedance Spectroscopy (EIS) over a range of state of charges (SoCs). Fig. 4a and b display the Nyquist plots derived from the EIS tests for both pairs for a number of SOCs before disassembly. In line with the literature, the shape of the Nyquist plots obtained is divided into 4 regions, the very high frequency region represents the inductance of cell and cables, the intersection of the inductive trail with the real axis corresponds with the electrolyte and connection resistances, a semicircle with radius $r$ governed by the chargetransfer phenomenon, and the low frequency region denotes the diffusion in the electrodes. Fig. $4 \mathrm{a}$ and $\mathrm{b}$ show that the plot is observed shifting to the right as the SoC decreases, which can be clearly seen at $20 \%$ and $100 \%$ SoC in both pairs. To highlight the influence of interconnections (busbars) on the impedance spectra, the EIS measurements at $60 \%$ SoC (nominal voltage) are chosen and displayed in Fig. 5. The impedance spectra of pair 2 has a noticeable shift to the right and longer inductive trail compared to pair1. This shift is dominated by the evolution in the total ohmic resistance of the system, which represents the electrolyte resistance, the contact resistance, and the electronic contacts. Furthermore, the response in medium and low frequency region look very similar for both pairs. The total ohmic resistance along with other dynamical parameters for each pair is then identified via fitting the EIS response to an ECM as shown in Fig.6. In this model, $L_{l}$ stands for the inductance of the pair and cables, $R_{l}$ (high-frequency resistance) denotes the electrolyte and connection resistances, and the $R_{2} \| C_{2}$ parallel circuit models the charge-transfer phenomenon. Therefore, the frequencydependent impedance expression for the ECM is introduced

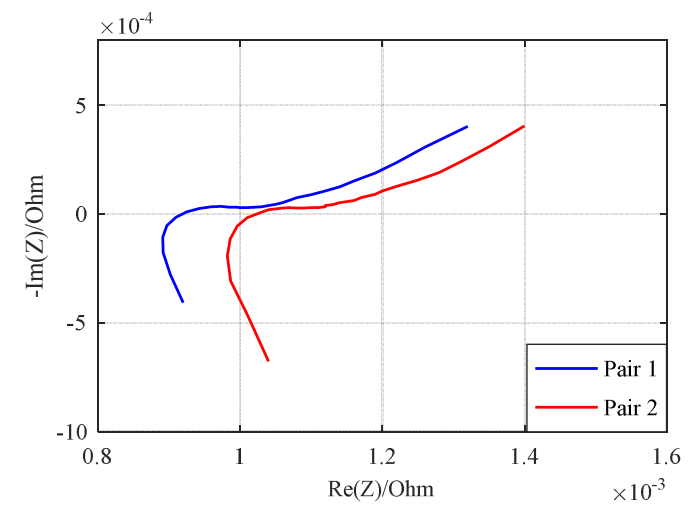

Fig. 5. Nyquist plot for parallel cells in Leaf module at $60 \% \mathrm{SoC}$.

as:

$$
Z(f)=L_{1} j 2 \pi f+R_{1}+\frac{R_{2}}{1+j 2 \pi f R_{2} C_{2}}
$$

where $j$ is the imaginary unit and $f$ is the frequency in Hertz. The diffusion part is excluded from the fitting process due to the impedance at this point is used as a metric to quantify cell ageing which is not considered in this research. Hence, high and medium frequencies ( 300 to $4 \mathrm{~Hz}$ ) are used to fit the EIS data using the ECM shown in Fig.6. Table. I. shows that there is a difference in the values of the resistances $R_{l}$, and $R_{2}$ for both pairs. Even though the capacity of pair 2 is higher than in pair 1 , the ohmic resistance $R_{I}$ of pair 1 is smaller which can be justified by considering the length of the copper busbar connecting the cells in pair 2 . This indicates that the increase in ohmic resistance cannot be always correlated with the capacity fade in the paralleled cell configurations due to interconnections. Preferably, the contribution of interconnections in total ohmic resistance would be zero, or at least negligible relative to cell impedance [3]. To determine this contribution in Nissan leaf module, the same impedance test is repeated for all pouch cells in the module. Fig.7a. and b display the Nyquist plot at $60 \%$ SoC for the four pouch cells. Obviously, the impedance at high frequency $(1 \mathrm{kHz})$ region is significantly reduced for each cell compared to the pairs. This is due to the contribution of busbar's inductance which dominates the Nyquist plot at this region as the total inductance of parallel cells is diminished. Moreover, in comparison with the pairs, the intersection point with the real axis for all cells is increased by around $50 \%$ to the right. From Table II it can be noticed that the average ohmic resistance for all cells is $1.5 \mathrm{~m} \Omega$, moreover, the charge transfer resistance values show a very small difference between cells. For instance, $R_{1 \text { Cell4 }}-R_{1 \text { Cell } 3}=1.61-1.44=0.17 \mathrm{~m} \Omega$ whereas both cells have the same capacity $(27.8 \mathrm{Ah})$. With the same variations in the ohmic resistances, the capacities of cell1 and cell 2 differ by $0.3 \mathrm{Ah}$.

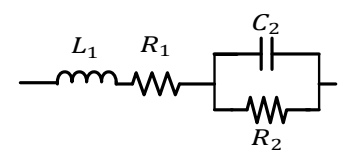

Fig. 6. Equivalent Circuit Model for Li Ion Battery. 
TABLE I. MODEL PARAMETERS

\begin{tabular}{|c|c|c|}
\hline Pair & $\boldsymbol{R}_{1} / \boldsymbol{m} \boldsymbol{\Omega}$ & $\boldsymbol{R}_{2} / \boldsymbol{m} \boldsymbol{\Omega}$ \\
\hline Pair 1 & 0.91 & 0.17 \\
\hline Pair 2 & 1.04 & 0.15 \\
\hline
\end{tabular}

These results demonstrate that the cell-to-cell variations in both pairs can be neglected in terms of impedance and capacity, and confirm that the capacity loss in the parallel connection is due to cell interconnections within the module.

\section{CONCLUSIONS AND FUTURE WORK}

Discharge capacity is a key feature that influences the EoL decision and hence the maintenance and replacement policy of a battery pack. The capacity loss due to parameter variations of in-pack cells has been addressed and extensively discussed in the literature. However, this paper demonstrates that the capacity loss in cells connected in parallel with no, or small variations between cells can reach up $6 \%$ in a retired battery pack available for second-life applications. This loss in capacity is caused by electrical contact resistances present in the inter-connects between cells forming the battery module. Future studies could evaluate the effect of interconnections on the ageing behaviour of the battery module in a second life application Moreover, different types of battery modules with a wide range of $\mathrm{SoH}$ will be tested and compared in terms of welding/joining techniques and busbar materials
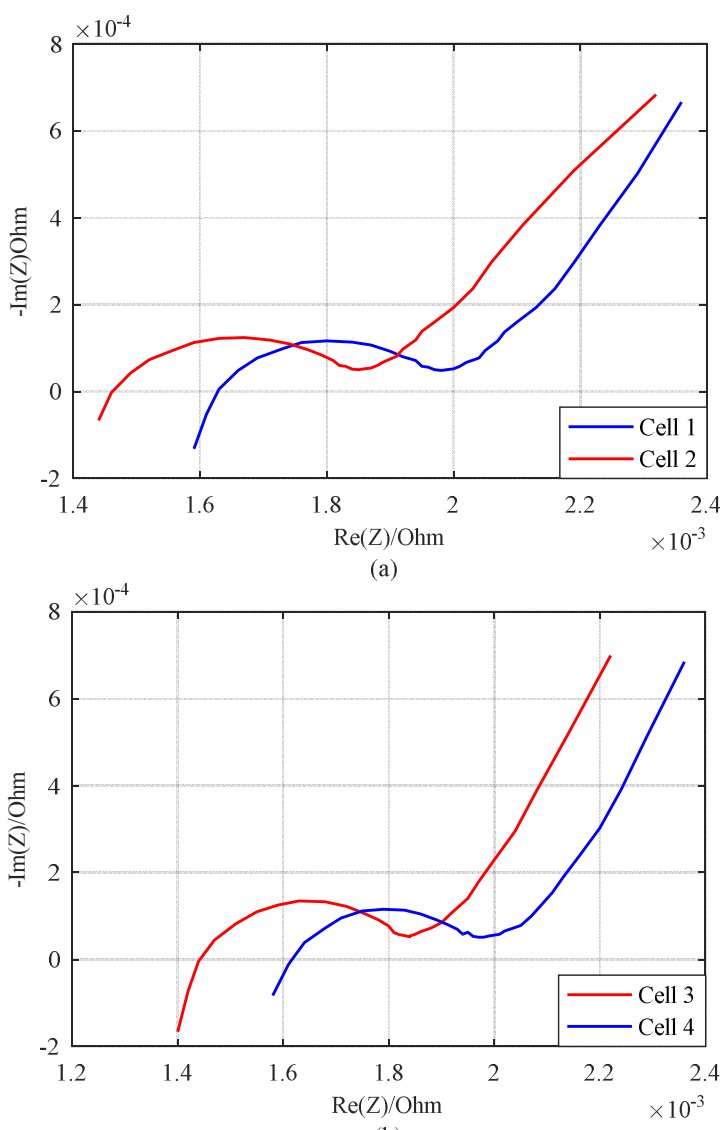

Fig. 7. Nyquist plot for individual cells in Leaf module at $60 \%$ SoC. (a) Cells in pair 1. (b) Cells in pair2.
TABLE II. MODEL PARAMETERS

\begin{tabular}{|c|c|c|}
\hline Cell & $\boldsymbol{R}_{1} / \boldsymbol{m} \boldsymbol{\Omega}$ & $\boldsymbol{R}_{2} / \boldsymbol{m} \boldsymbol{\Omega}$ \\
\hline Cell 1 & 1.46 & 0.36 \\
\hline Cell 2 & 1.62 & 0.34 \\
\hline Cell 3 & 1.44 & 0.38 \\
\hline Cell 4 & 1.61 & 0.37 \\
\hline
\end{tabular}

\section{ACKNOWLEDGMENT}

The authors acknowledge the financial support of the Faraday Institution: ISCF Faraday Challenge Fast Start Project on "Recycling and Reuse of lithium ion batteries" made available through "EP/FIRG005".

\section{REFERENCES}

[1] E. Hosseinzadeh, J. Marco, and P. Jennings, "Combined electrical and electrochemical-thermal model of parallel connected large format pouch cells," J. Energy Storage, vol. 22, pp. 194-207, Apr. 2019.

[2] E. Martinez-Laserna, E. Sarasketa-Zabala, I. V. Sarria, D. I. Stroe, M. Swierczynski, A. Warnecke, J.-M. Timmermans, S. Goutam, N. Omar, and P. Rodriguez, "Technical viability of battery second life: A study from the ageing perspective," IEEE Trans. Ind. Appl., vol. 54, no. 3, pp. 2703-2713, May/Jun. 2018.

[3] T. Bruen and J. Marco, "Modelling and experimental evaluation of parallel connected lithium ion cells for an electric vehicle battery system," J. Power Sources, vol. 310, pp. 91-101, Apr. 2016.

[4] B. K. Sovacool, J.-C. Rogge, C. Saleta, and E. Masterson-Cox, "Transformative versus conservative automotive innovation styles: Contrasting the electric vehicle manufacturing strategies for the BMW i3 and Fiat 500e," Environ. Innov. Soc. Trans, Feb. 2019.

[5] G. Reid and J. Julve, "Second life-batteries as flexible storage for renewables energies," Bundesverband Erneuerbare Energie eV (BEE), 2016

[6] A. Fill, S. Koch, and K. P. Birke, "Analytical model of the current distribution of parallel-connected battery cells and strings," J. Energy Storage, vol. 23, pp. 37-43, Jun. 2019.

[7] M. Baumann, L. Wildfeuer, S. Rohr, and M. Lienkamp, "Parameter variations within Li-Ion battery packs - Theoretical investigations and experimental quantification," J. Energy Storage, vol. 18, pp. 295-307, Aug. 2018.

[8] G. J. Offer, V. Yufit, D. A. Howey, B. Wu, and N. P. Brandon, "Module design and fault diagnosis in electric vehicle batteries," J. Power Sources, vol. 206, pp. 383-392, May. 2012. 